\title{
Changes in flagellar bending during the course of hyperactivation in hamster spermatozoa
}

\author{
M. Kinukawa, M. Nagata and F. Aoki* \\ Department of Integrated Biosciences, Graduate School of Frontier Sciences, \\ University of Tokyo, Kashiwa City, Chiba 277-8562, Japan
}

The motility pattern of mammalian spermatozoa changes during migration in the female genital tract and during incubation in vitro. This change in motility is termed hyperactivation. Hyperactivated spermatozoa swim vigorously in 'whiplash', 'figure-8' or 'small circle' trajectories. In this study, a quantitative analysis was carried out of the changes in the motility pattern of hamster spermatozoa during incubation to investigate the mechanism regulating hyperactivation. In the culture system used in this study, hyperactivation occurred $4 \mathrm{~h}$ after incubation. Several parameters in the analysis of sperm movement pattern were examined. Curvilinear velocity, average path velocity and straightness abruptly increased between 2 and $4 \mathrm{~h}$. However, linearity, amplitude of lateral head displacement, beat cross frequency and average wavelength gradually changed with time. In the analysis of flagellar bending, the bend angles were measured after dividing images of the flagellum into short lengths. Flagellar bending changed in different manners in each region during incubation. The asymmetry in the direction of the curve of the head gradually increased with time in the first half of the flagellum. The flexibility, which was determined using the amplitude of bending and the rate of change in bend angles, abruptly decreased between $10 \mathrm{~min}$ and $1 \mathrm{~h}$, and then increased between 2 and $4 \mathrm{~h}$ in the first half of flagellum. These results indicate that complex physiological changes occur before hyperactivation.

\section{Introduction}

The motility pattern of mammalian spermatozoa changes before fertilization. Freshly ejaculated mammalian spermatozoa are actively motile, but are unable to fertilize an oocyte. Spermatozoa need to spend a period of time in either the female reproductive tract or an appropriate in vitro environment to become competent for fertilization. During this period, spermatozoa undergo a physiological change termed capacitation. This is a prerequisite for the acrosome reaction (Yanagimachi, 1994). At the same time as spermatozoa undergo capacitation in vitro, their swimming pattern changes. They swim more vigorously than before, in 'whiplash', 'figure-8' or 'small circle' trajectories. This change in sperm motility around the time of capacitation seems to be common in mammalian species, and has been termed hyperactivation (Yanagimachi, 1981). A common feature of hyperactivated sperm motility in all species examined is that the spermatozoa do not swim in a straight line: in some species they swim in a zig-zag trajectory and in others they swim in small circles. In microscopic observations, the change to the hyperactivated motility pattern seems to occur abruptly (Yanagimachi, 1970),

*Correspondence

Email: aokif@k.u-tokyo.ac.jp although in hamster spermatozoa the ratio for several motility patterns of planar, circular, helical and hatchet changed over time (Shivaji et al., 1995). The motility of hyperactivated spermatozoa has been analysed using computer assisted sperm analysis (CASA) in various species, for example mice (Neill and Olds-Clarke, 1987), humans (Mortimer and Swan, 1995), sheep (Mortimer and Maxwell, 1999) and rats (Cancel et al., 2000). In CASA analysis, these patterns have been compared in freshly collected and hyperactivated spermatozoa, but have not been examined over time, as hyperactivation appears to occur abruptly. However, in elucidating the mechanism of hyperactivation, it is important to examine the time-dependent changes in these parameters.

For an understanding of the mechanism of hyperactivation, analysis of flagellar bending is more important than that of swimming patterns, since flagellar bending reflects the microtubule sliding that generates the power of movement. However, most CASA analyses have measured only parameters involved in swimming patterns, and not flagellar bending. Some studies have analysed flagellar bending and its relationship to the swimming pattern in hyperactivated spermatozoa. Aoki et al. (1994) analysed the bend angles of the flagellum in hamster spermatozoa and showed that flagellar asymmetry increased after $4 \mathrm{~h}$ of in vitro culture. The angles of the bends in the same 
direction as the curve of the hook-shaped head increased in all regions of the flagellum, whereas those of the bends in the opposite direction did not. Ishijima et al. (2002) also reported that hyperactivated spermatozoa have remarkably asymmetrical flagellar waves of large amplitude. However, no reports have described the timedependent change in flagellar bending in the course of hyperactivation.

The mechanism that brings about hyperactivation remains unknown. It has been suggested that calcium and cyclic nucleotides play important roles in the regulation of sperm motility. Extracellular $\mathrm{Ca}^{2+}$ is required to maintain the hyperactivated motility pattern of hamster spermatozoa in vitro (Yanagimachi, 1994). An increased intracellular cAMP concentration is associated with the expression of hyperactivated motility (White and Aitken, 1989). In a de-membranated sperm model, a movement pattern resembling hyperactivated motility was observed when both $\mathrm{Ca}^{2+}$ and cAMP were added (Lindemann et al., 1991). Although these substances seem to be involved, little is known about the mechanism regulating hyperactivation.

Proteins that may regulate sperm motility have been found to be localized in the sperm flagellum. Cyclic nucleotide-gated channels control $\mathrm{Ca}^{2+}$ entry into bull spermatozoa. The $\alpha$ subunit is observed along the entire flagellum, whereas the short $\beta$ subunit is localized on the principal piece of the flagellum (Wiesner et al., 1998). CatSper, which is a putative sperm cation channel, regulates the CAMP-mediated $\mathrm{Ca}^{2+}$ influx and motility in mouse spermatozoa. CatSper is localized in the principal piece of the flagellum (Ren et al., 2001). Protein kinase A regulates sperm motility through the cAMP-dependent phosphorylation of proteins. A-kinase anchoring protein is localized to the principal piece of the flagellum in the region of fibrous sheath in mouse spermatozoa (Carrera et al., 1994), bull spermatozoa (Moss et al., 1999), and hamster spermatozoa (Kula Nand and Shivaji, 2002). These facts indicate that the regulation of flagellar bending differs in different parts of the flagellum.

In the present study, motility patterns and flagellar bending were analysed by measuring various parameters to investigate the mechanism underlying hyperactivation in hamster spermatozoa cultured in vitro. The procedure used two novel approaches. First, the time-dependent changes in the parameters examined during in vitro culture were investigated. Second, images of the flagellum were divided into small regions to examine local changes in bending.

\section{Materials and Methods}

\section{Media preparation}

The medium used for in vitro culture of hamster spermatozoa was developed by Suarez (1988) to maximize the occurrence of hyperactivation and mini- mize the acrosome reaction. The medium consisted of $110 \mathrm{mmol} \mathrm{NaCl} \mathrm{I}-1,5.0 \mathrm{mmol} \mathrm{KCl} \mathrm{I}{ }^{-1}, 2.4 \mathrm{mmol} \mathrm{CaCl}_{2}$ $\mathrm{I}^{-1}, 0.49 \mathrm{mmol} \mathrm{MgCl}_{2} \mathrm{I}^{-1}, 0.36 \mathrm{mmol} \mathrm{NaH}_{2} \mathrm{PO}_{4} \mathrm{I}^{-1}$, $24.9 \mathrm{mmol} \mathrm{NaHCO}_{3} \mathrm{I}^{-1}, 25 \mathrm{mmol}$ Hepes (N-2hydroxyethyl-piperazine- $\mathrm{N}$-2-ethane sulphonic acid) $\mathrm{I}^{-1}$, $6.25 \mathrm{mmol}$ lactic acid $\mathrm{I}^{-1}, 0.125 \mathrm{mmol}$ sodium pyruvate $\mathrm{I}^{-1}, 0.5 \mathrm{mmol}$ hypotaurine $\mathrm{I}^{-1}, 5.0 \mathrm{mmol}$ glucose $\mathrm{I}^{-1}$, $12 \mathrm{mg}$ bovine serum albumin (BSA; fatty acid-free fraction V, Sigma Chemical Inc., Tokyo) $\mathrm{ml}^{-1}, 100 \mathrm{U}$ penicillin $\mathrm{ml}^{-1}$ and $0.1 \mathrm{mg}$ streptomycin $\mathrm{ml}^{-1}$. The $\mathrm{pH}$ was adjusted to 7.4 .

\section{Sperm preparation}

Male golden hamsters (Mesocricetus auratus) were raised and maintained in a light-controlled room $(12 \mathrm{~h}$ light: $12 \mathrm{~h}$ dark $)$ at a constant temperature $\left(22^{\circ} \mathrm{C} \pm 1^{\circ} \mathrm{C}\right)$. Sexually mature hamsters were killed by chloroform inhalation and the cauda epididymidis was promptly removed. After removing any blood from the epididymal surface with physiological salt solution, the distal tubules were punctured with an 18-gauge needle in five to ten places, and a mass of spermatozoa squeezed out with forceps into a plastic Petri dish $(35 \mathrm{~mm} \times 10 \mathrm{~mm})$ containing $5 \mathrm{ml}$ of medium pre-warmed to $37^{\circ} \mathrm{C}$. The spermatozoa were incubated at $37^{\circ} \mathrm{C}$ under $5 \% \mathrm{CO}_{2}$ in air.

\section{Sample preparation}

Aliquots $(14 \mu \mathrm{l})$ of sperm suspension were withdrawn to analyse the movement pattern and flagellar bending after $10 \mathrm{~min}$ and 1,2 and $4 \mathrm{~h}$ of incubation. The suspension was diluted with medium to give a final concentration of about $2 \times 10^{6}$ spermatozoa $\mathrm{ml}^{-1}$. A glass slide, coated with $0.5 \%(\mathrm{w} / \mathrm{v})$ PVP (polyvinylpyrrolidone K-90) to minimize the adhesion of spermatozoa to the glass surfaces, was warmed to $37^{\circ} \mathrm{C}$. The suspension was placed on the glass slide and covered with an $18 \mathrm{~mm} \times 24 \mathrm{~mm}$ cover slip to make a depth of about $32 \mu \mathrm{m}$. As soon as a sample was prepared, photographs were taken for analysis.

\section{Analysis of the pattern of sperm movement}

The pattern of sperm movement was analysed by taking photographs at 30 frames $\mathrm{s}^{-1}$ with a FASTCAMNet high-speed camera (PHOTRON Ltd., Tokyo) and the images obtained were recorded and analysed using Movie Ruler (PHOTRON). The analysis was performed using 30 successive frames, and three successive frames were used for the analysis of the number of bends. The exposure time was 1:2000 s under a phase-contrast microscope. Motile spermatozoa were distinguished from immotile spermatozoa as those that were located at different positions in successive frames. Motile spermatozoa were selected for analysis at random, whereas immotile spermatozoa were omitted from 
(a)

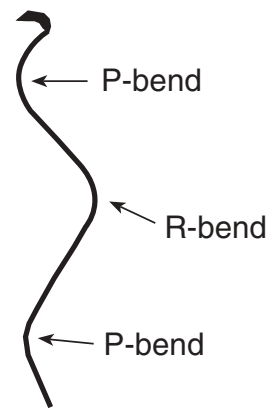

(b)

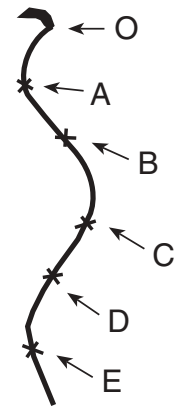

(c)

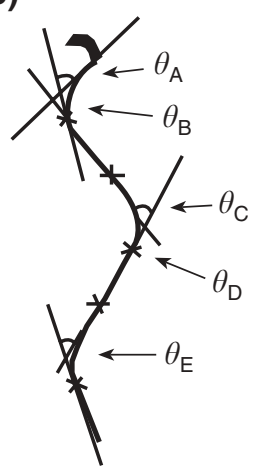

Fig. 1. Procedure used to analyse flagellar bending in hamster spermatozoa. (a) Bends in the same direction as the curve of the hook-shaped head were defined as reverse bends (R-bends) and those in the opposite direction were defined as principal bends ( $\mathrm{P}$ bends). (b) The points on the flagellum 30, 60, 90, 120 and $150 \mu \mathrm{m}$ from the head-midpiece junction $(\mathrm{O})$ were designated as $\mathrm{A}, \mathrm{B}, \mathrm{C}$, $D$ and $E$, respectively. (c) The bend angles were measured as the angles between consecutive tangents. The regional angles between $O$ and $A, A$ and $B, B$ and $C, C$ and $D$, and $D$ and $E$ were termed $\theta_{A}$, $\theta_{B}, \theta_{C}, \theta_{D}$ and $\theta_{E}$, respectively. R-bends were assigned plus values and $\mathrm{P}$-bends were assigned minus values.

analysis. In the analysis, the swimming trajectories of spermatozoa were determined by tracing the sequential positions of the head-midpiece junction. The sperm motility parameters determined in this study were: (1) VSL (straight-line velocity), the distance between the first- and last-tracked points divided by the time elapsed; (2) VCL (curvilinear velocity), the sum of the distances between adjacent points divided by the time elapsed; (3) VAP (average path velocity), a smoothing path of the average of five successive points divided by the time elapsed, which reduced the effect of lateral head displacement; (4) LIN (linearity), an index of the straightness of the path, given by VSL/VCL $\times 100$; (5) STR (straightness), an index of the departure of the sperm path from a straight line, given by VSL/VAP $\times 100$; (6) ALH (amplitude of lateral head displacement), an index of lateral head displacement, given by the maximum value of the distance of any point on the track from the corresponding average path, multiplied by two; (7) beat cross frequency, an index of vigour, given by the frequency with which the cell track crosses the smoothing path in either direction; cell track and smoothing path were determined in the measurement of VCL and VAP, respectively (Cancel et al., 2000); and (8) average wavelength, an index of wavelength, derived from the length of the flagellum (180 $\mu \mathrm{m}$; Woolley, 1977) divided by the number of bends.

\section{Analysis of flagellar bending}

Flagellar bending of spermatozoa was analysed by taking photographs at 250 frames per $3 \mathrm{~s}$ with a (a)

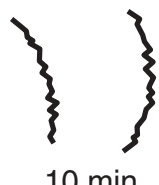

$10 \mathrm{~min}$

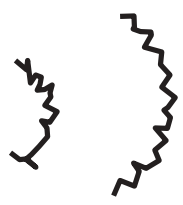

$2 \mathrm{~h}$

(b)

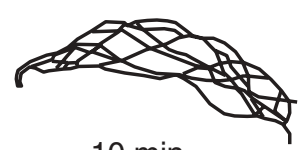

$10 \min$

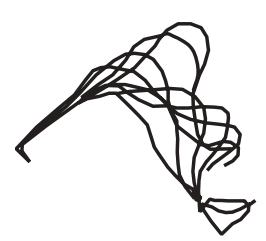

$2 \mathrm{~h}$

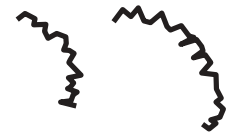

$1 \mathrm{~h}$

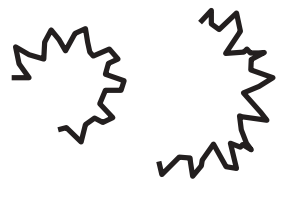

$4 \mathrm{~h}$
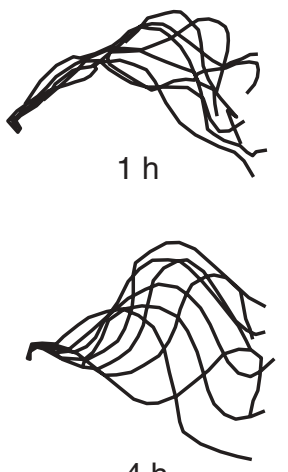

$4 \mathrm{~h}$
Fig. 2. (a) Typical movement trajectories of hamster spermatozoa. Photographs were taken at 30 frames $\mathrm{s}^{-1}$. The dots show the location of the head-midpiece junction in 30 successive frames. The spermatozoa were incubated for $10 \mathrm{~min}$ and 1, 2 and $4 \mathrm{~h}$. (b) Typical patterns of flagellar bending. Photographs were taken at 250 frames per $3 \mathrm{~s}$. Images of spermatozoa were traced in eight successive frames after superimposing the axes of the heads. The spermatozoa were incubated for $10 \mathrm{~min}$ and 1,2 and $4 \mathrm{~h}$.

FASTCAM-Net high-speed camera (PHOTRON) and the images obtained were recorded and analysed using Movie Ruler (PHOTRON). The analysis was performed using eight successive frames. The exposure time was 1:2000 s under a phase-contrast microscope. The direction of each bend was determined according to the direction of hook-shaped projection of head as described by Woolley (1977). A bend in the same direction as the curve of the hook-shaped head was defined as a reverse bend ( $\mathrm{R}$-bend) and one in the opposite direction was defined as a principal bend (P-bend). Reverse bends were assigned a plus value and principal bends a minus value (Fig. 1a). To measure regional bend angles in the flagellum, each image of a flagellum was divided into $30 \mu \mathrm{m}$ sections from the head-midpiece junction. The points that were $0,30,60,90,120$ and $150 \mu \mathrm{m}$ from the head-midpiece junction were termed points $\mathrm{O}, \mathrm{A}, \mathrm{B}, \mathrm{C}$, $D$ and $E$, respectively (Fig. 1b). Tangent lines were drawn at each point. The regional bend angle was measured as the supplementary angle formed by two successive 
(a)

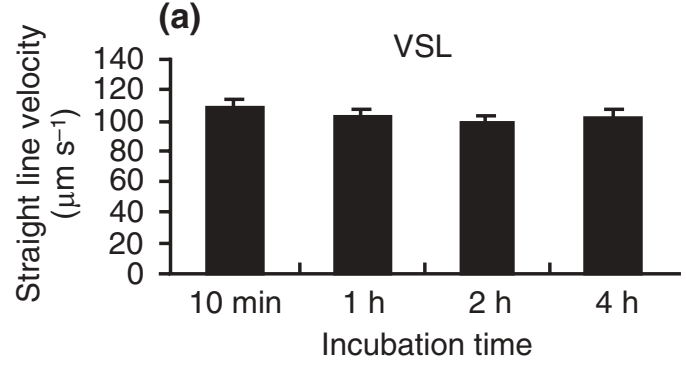

(c)

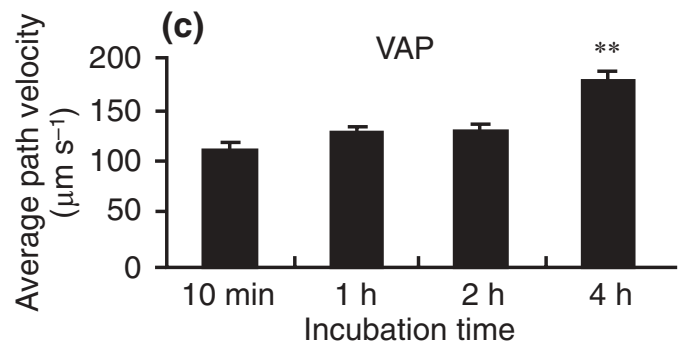

(e)

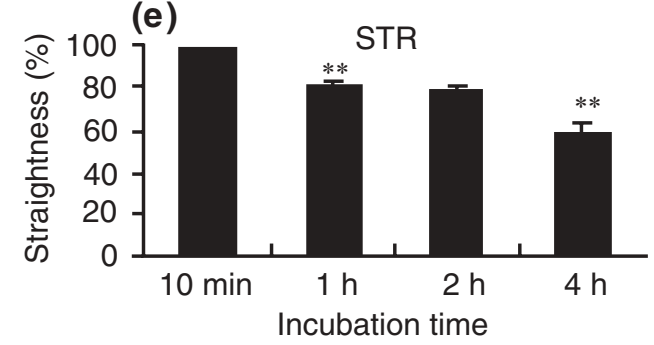

(b)

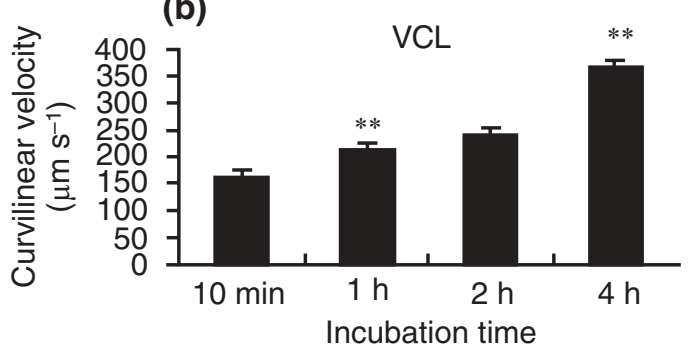

(d)
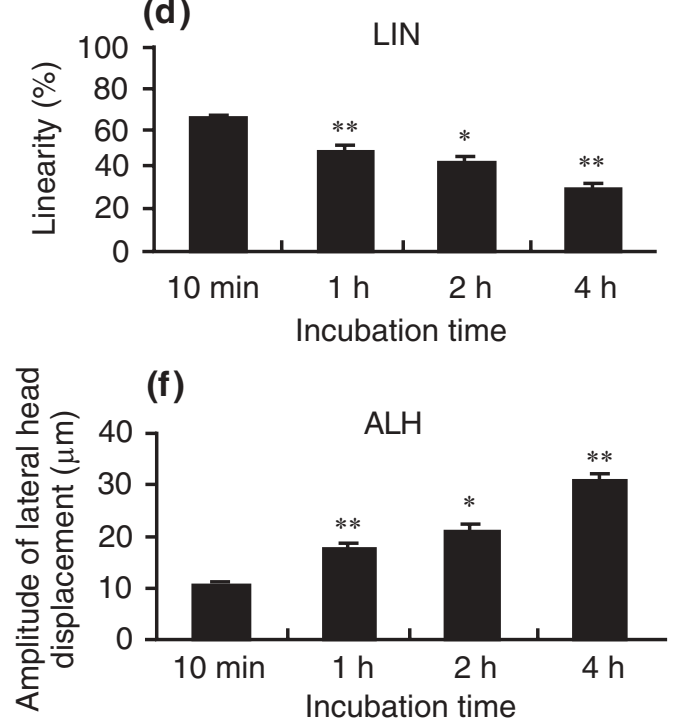

Fig. 3. Analysis of the movement pattern of hamster spermatozoa using various parameters. Photographs were taken at 30 frames $\mathrm{s}^{-1}$. The analysis used 30 successive frames. (a) VSL, straight-line velocity; (b) VCL, curvilinear velocity; (c) VAP, average path velocity; (d) LIN, linearity; (e) STR, straightness; and (f) ALH, amplitude of lateral head displacement. The experiments were performed three times. The data were pooled and expressed as the mean \pm SEM $(n=18)$. Asterisks indicate significant differences from the value at the previous time point (Student's $t$ test; ${ }^{*} P<0.05$ and $* * P<0.01)$.

tangents. The angles between the tangent lines to $\mathrm{O}$ and $\mathrm{A}, \mathrm{A}$ and $\mathrm{B}, \mathrm{B}$ and $\mathrm{C}, \mathrm{C}$ and $\mathrm{D}$, and $\mathrm{D}$ and $\mathrm{E}$ were termed $\theta_{A}, \theta_{B}, \theta_{C}, \theta_{D}$ and $\theta_{E}$, respectively (Fig. $1 \mathrm{C}$ ). In eight successive frames, the maximum and minimum of each regional bend angle, the difference between the maximum and minimum, the average of the maximum and minimum, and the sum of $\theta_{\mathrm{A}}-\theta_{\mathrm{E}}$ were determined. In addition, the rate of change of flagellar bending was examined by determining the changes in each regional bend angle between two successive frames.

\section{Results}

\section{Analysis of the movement pattern}

The changes in motility pattern before hyperactivation were clarified by analysing the change in the movement pattern after incubation for $10 \mathrm{~min}$ and 1,2 and $4 \mathrm{~h}$. After incubation for $10 \mathrm{~min}$, the spermatozoa swam in a plane without rolling and followed a circular trajectory, in which the hooks of the heads were always directed into the circles. The circular trajectory became much smaller with time until $4 \mathrm{~h}$ (Fig. 2a). The track of lateral movement also increased in length with time. These characteristics of the changes in movement pattern were also shown quantitatively by measuring the parameters VSL, VCL, VAP, LIN, STR and ALH (Fig 3) and beat cross frequency and average wavelength (Fig. 4). $\mathrm{VCL}$, an index of swimming speed, increased slightly until $2 \mathrm{~h}$, and then increased markedly between 2 and $4 \mathrm{~h}$ (Fig. 3b). VAP, obtained by measuring the smooth path of the average of five successive points to reduce the effect of lateral head displacement, also increased slightly but not significantly until $2 \mathrm{~h}$ and then increased markedly between 2 and $4 \mathrm{~h}$ (Fig. 3c). LIN, an index of path straightness, decreased with time, reflecting the time-dependent decrease in the radius of the circular swimming path or the increase in lateral head displacement (Fig. 3d). STR, which is an index of path straightness that excludes the effect of lateral head 


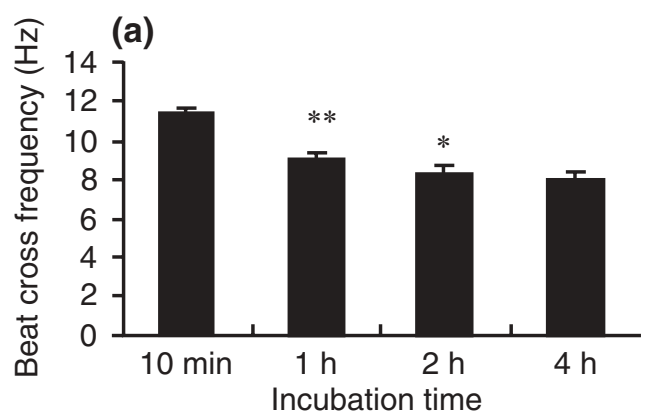

(b)

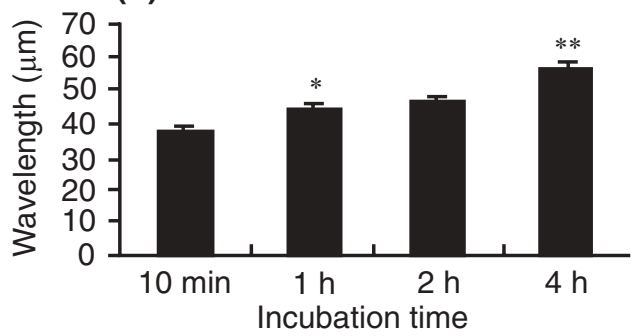

Fig. 4. Analysis of (a) beat cross frequency and (b) average wavelength of hamster spermatozoa. Photographs were taken at 30 frames s$~^{-1}$. The analysis used 30 successive frames. The experiments were performed three times. The data were pooled and expressed as the mean $\pm \operatorname{SEM}(n=18)$. Asterisks indicate significant differences from the value at the previous time point (Student's $t$ test; ${ }^{*} P<0.05$ and ${ }^{* *} P<0.01$ ).

displacement, decreased markedly between 1 and $2 \mathrm{~h}$, and between 2 and $4 \mathrm{~h}$, indicating that the swimming path became smaller in two steps (Fig. 3e). ALH, an index of lateral head displacement, gradually increased with time, reflecting the time-dependent increase in lateral head displacement (Fig. 3f).

\section{Measuring beat cross frequency and wavelength}

The beat cross frequency decreased markedly between $10 \mathrm{~min}$ and $1 \mathrm{~h}$, and slightly between 1 and $2 \mathrm{~h}$. It did not change between 2 and $4 \mathrm{~h}$ (Fig. $4 \mathrm{a}$ ). In contrast, the wavelength increased with time (Fig. 4b); it increased between $10 \mathrm{~min}$ and $1 \mathrm{~h}$, slightly but not significantly between 1 and $2 \mathrm{~h}$, and then markedly between 2 and $4 \mathrm{~h}$.

\section{Analysis of flagellar bending}

Changes in flagellar bending were analysed by measuring the bend angles in different regions, as the bending would change in different manners in each region during incubation. The changes in the average bend angles for each region O-A through to $D-E\left(\theta_{A}-\theta_{E}\right)$ (Fig. 5a), show that in all regions, the values increased between $10 \mathrm{~min}$ and $1 \mathrm{~h}$, indicating an increase in the
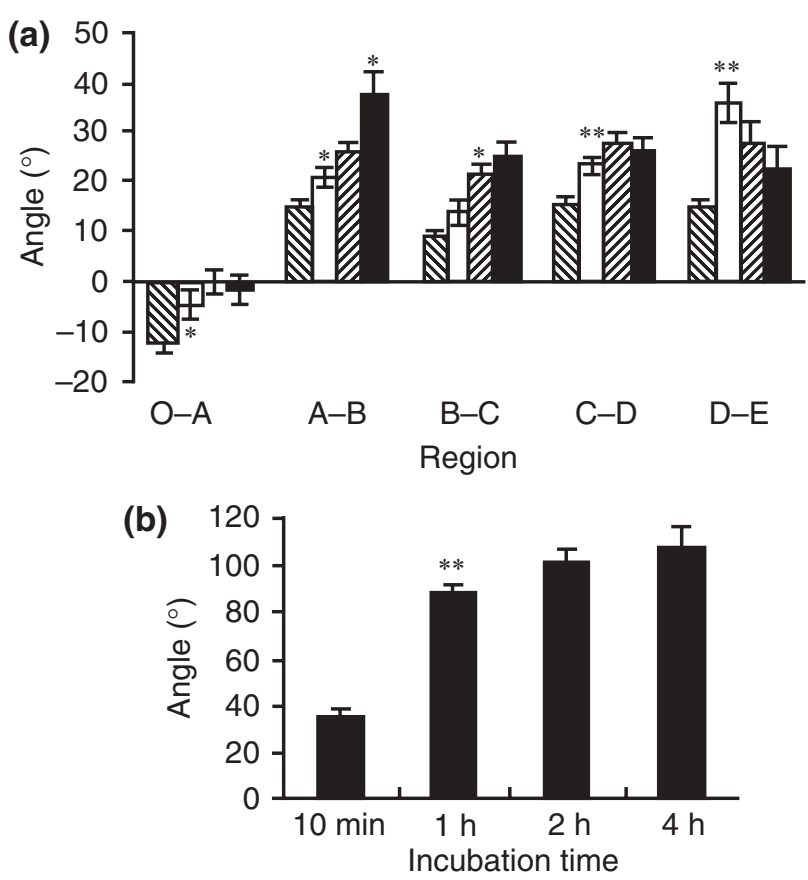

Fig. 5. Analysis of flagellar bending in hamster spermatozoa determining (a) the average bend angle in each flagellar region (at $\mathbb{\$}: 10 \mathrm{~min} ; \square: 1 \mathrm{~h} ; 2 \mathrm{~h}$; and $\square: 4 \mathrm{~h}$ ), and (b) the sum of the average bend angles for each region. Photographs were taken at 250 frames per $3 \mathrm{~s}$. The analysis used eight successive frames. The regional bend angles in the flagellum were measured by designating the points on the flagellum 30,60, 90, 120 and $150 \mu \mathrm{m}$ from the head-midpiece junction $(\mathrm{O})$ as $\mathrm{A}, \mathrm{B}, \mathrm{C}, \mathrm{D}$ and $E$, respectively. The regions between $O$ and $A, A$ and $B, B$ and $\mathrm{C}, \mathrm{C}$ and $\mathrm{D}$, and $\mathrm{D}$ and $\mathrm{E}$ were termed $\mathrm{O}-\mathrm{A}, \mathrm{A}-\mathrm{B}, \mathrm{B}-\mathrm{C}$, $C-D$ and $D-E$, respectively. The experiments were performed three times. The data were pooled and expressed as the mean \pm SEM $(n=21)$. Asterisks indicate significant differences from the value at the previous time point (Student's $t$ test; ${ }^{*} P<0.05$ and ** $P<0.01)$.

asymmetry of the direction of the R-bend during this period. The values of $\theta_{A}, \theta_{B}$ and $\theta_{C}$ gradually increased with time until $4 \mathrm{~h}$, indicating that the asymmetry of the direction of the R-bend increased continuously in the first half of the flagellum. The value of $\theta_{B}$ in region $\mathrm{A}-\mathrm{B}$, the region generating the dominant second bend, increased markedly between 2 and $4 \mathrm{~h}$. However, $\theta_{\mathrm{E}}$ increased between $10 \mathrm{~min}$ and $1 \mathrm{~h}$ and then decreased until $4 \mathrm{~h}$. Thus, the bend angles in each region changed differently. The bend angles in all regions were summed to analyse the total change in the asymmetry of bending in the entire flagellum. The sum of $\theta_{\mathrm{A}}-\theta_{\mathrm{E}}$ increased markedly between $10 \mathrm{~min}$ and $1 \mathrm{~h}$ and then increased gradually, but not significantly, until $4 \mathrm{~h}$ (Fig. 5b).

The maximum and minimum bend angles in eight successive frames in each region were determined. Asymmetry can be evaluated from these values, as well as from the average bend angles discussed above. 

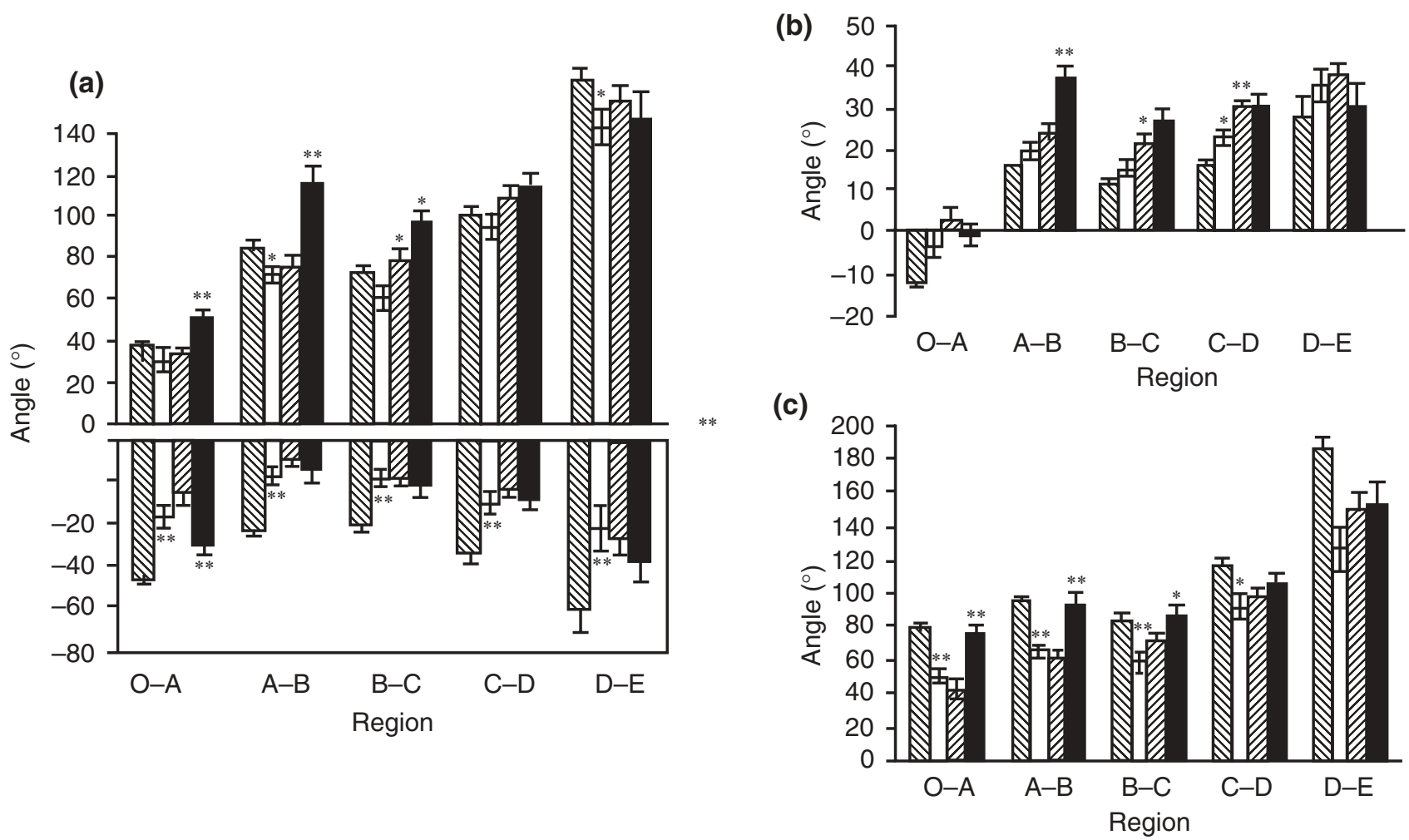

Fig. 6. Analysis of flagellar bending in hamster spermatozoa based on the maximum and minimum bend angles in each flagellar region at $\mathbb{\$}: 10 \mathrm{~min} ; \square: 1 \mathrm{~h} ; \mathbf{2}: 2 \mathrm{~h}$; and $\mathbf{\square}: 4 \mathrm{~h}$. Photographs were taken at 250 frames per $3 \mathrm{~s}$. The analysis used eight successive frames. (a) The maximum and minimum regional bend angles; (b) the average of the maximum and minimum; and (c) the difference between the maximum and minimum. The points on the flagellum 30,60, 90, 120 and $150 \mu \mathrm{m}$ from the head-midpiece junction (O) were designated as A, B, C, D and E, respectively. The regions between $O$ and $A, A$ and $B, B$ and $C, C$ and $D$, and D and E were termed $O-A$, A-B, B-C, C-D and D-E, respectively. The experiments were performed three times. The data were pooled and expressed as the mean \pm SEM $(n=21)$. Asterisks indicate significant differences from the value at the previous time point (Student's $t$ test; ${ }^{*} P<0.05$ and $* * P<0.01)$

In addition, these values allow evaluation of changes in flexibility. The maximum values decreased between $10 \mathrm{~min}$ and $1 \mathrm{~h}$ and then increased until $4 \mathrm{~h}$ in all regions of the flagellum except for the distal region, D-E (Fig. 6a). The changes in the minimum values were almost the reverse of those in the maximum values. The maximum and minimum values were averaged in each region to evaluate the asymmetry (Fig. 6b). The pattern of change in these values was similar to that in the average bend angles described above (Figs $5 \mathrm{a}$ and $6 \mathrm{~b}$ ); the average of maximum and minimum values gradually increased with time in the first half of the flagellum. The flexibility was evaluated using the difference between the maximum and minimum values $(M X-M N)$. MX-MN decreased prominently between $10 \mathrm{~min}$ and $1 \mathrm{~h}$ in all regions. Subsequently, at $4 \mathrm{~h}$, it abruptly increased to near the original value at $10 \mathrm{~min}$ in regions $\mathrm{O}-\mathrm{A}, \mathrm{A}-\mathrm{B}$ and $\mathrm{B}-\mathrm{C}$, but not in regions $\mathrm{C}-\mathrm{D}$ and $\mathrm{D}-\mathrm{E}$, indicating that the flexibility was decreased at $1 \mathrm{~h}$ in all regions of the flagellum, and then restored at $4 \mathrm{~h}$ in the first half of the flagellum, but not in the second half.
Although the changes in $M X-M N$ reflect changes in flexibility, this index is insufficient for evaluating the flexibility by itself; rather, it reflects amplitude. The flagellum would not appear to be flexible when the movement of the flagellum is slow, even if the amplitude is high. Therefore, the flexibility was evaluated by determining the changes in the bend angles between two successive frames (Fig. 7). These values represent the rates of change in flagellar bending. The values changed in a manner similar to the change in MX-MN (Figs 6c and 7 ). They decreased at $1 \mathrm{~h}$ in all regions and then abruptly increased at $4 \mathrm{~h}$ in regions $\mathrm{O}-\mathrm{A}$ and $\mathrm{A}-\mathrm{B}$. In region $\mathrm{B}-\mathrm{C}$, the values remained constant between 1 and $4 \mathrm{~h}$, whereas they decreased in regions $\mathrm{C}-\mathrm{D}$ and D-E. Combined with the value of MX-MN, the flexibility decreased at $1 \mathrm{~h}$ in all regions and then was abruptly restored at $4 \mathrm{~h}$ in the first half of the flagellum.

Typical patterns of flagellar bending, which has characteristic trajectories, changing with time, are shown (Fig. 2b) by tracing the movement of the flagellum. 


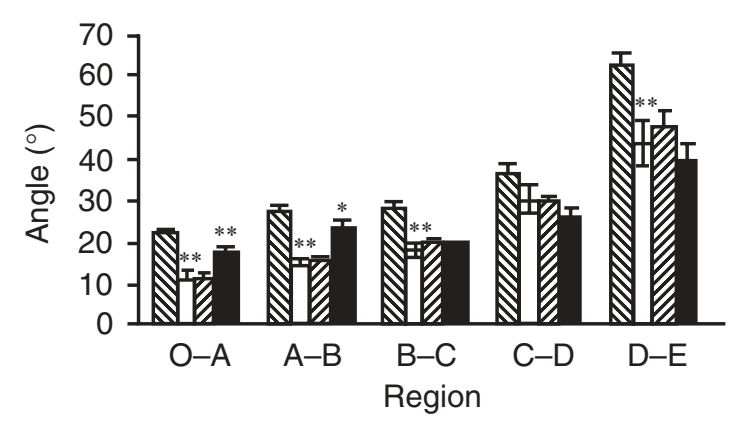

Fig. 7. Analysis of the changes in the bend angles in hamster spermatozoa between two successive frames in each region at $\mathbb{\$}: 10 \mathrm{~min} ; \square: 1 \mathrm{~h} ; \mathbf{Z}: 2 \mathrm{~h}$; and $\mathbf{\square}: 4 \mathrm{~h}$. Photographs were taken at 250 frames per $3 \mathrm{~s}$. The analysis used eight successive frames. The points on the flagellum 30,60, 90, 120 and $150 \mu \mathrm{m}$ from the head-midpiece junction $(\mathrm{O})$ were designated as $\mathrm{A}, \mathrm{B}, \mathrm{C}, \mathrm{D}$ and $\mathrm{E}$, respectively. The regions between $O$ and $A, A$ and $B, B$ and $C, C$ and $\mathrm{D}$, and $\mathrm{D}$ and $\mathrm{E}$ were termed $\mathrm{O}-\mathrm{A}, \mathrm{A}-\mathrm{B}, \mathrm{B}-\mathrm{C}, \mathrm{C}-\mathrm{D}$ and $\mathrm{D}-\mathrm{E}$, respectively. The experiments were performed three times. The data were pooled and expressed as the mean $\pm \operatorname{SEM}(n=21)$. Asterisks indicate significant differences from the value at the previous time point (Student's $t$ test; ${ }^{*} P<0.05$ and ${ }^{* *} P<0.01$ ).

\section{Discussion}

The present study developed two novel analytical measures to investigate changes in sperm motility patterns during the process of hyperactivation. First, the regional changes in flagellar bending were analysed by dividing images of the flagellum into short regions. Second, the changes in movement patterns and flagellar bending were examined with time of incubation. From these analyses, flagellar bending was found to change in a different manner in each region, and in a complex manner with time.

Analysing the regional changes in flagellar bending is important for investigating the mechanism of hyperactivation, as several reports indicate that regulation of flagellar bending differs regionally in the flagellum. Local iontophoretic application of ATP demonstrated that sliding occurs in each region independently (Shingyoji et al., 1977). The $\alpha$ subunit of cyclic nucleotide-gated channels is observed along the entire flagellum, whereas the short $\beta$ subunit is localized in the principal piece of the flagellum (Wiesner et al., 1998). CatSper, which is a putative sperm cation channel regulating cAMPmediated $\mathrm{Ca}^{2+}$ influx, is localized in the principal piece of the flagellum (Ren et al., 2001). The present study has demonstrated that the bending pattern changes in a different way in each region during the course of hyperactivation. The degree of asymmetry of the direction of the R-bend increased in the first half of the flagellum with time, but not in the second half. It increased markedly between 2 and $4 \mathrm{~h}$ in region A-B. This pattern of change corresponded to changes in the movement pattern. The circular trajectory of movement decreased with time and was much smaller between 2 and $4 \mathrm{~h}$.
In contrast, when the bend angles of all the regions were summed, the asymmetry increased prominently between $10 \mathrm{~min}$ and $1 \mathrm{~h}$. Therefore, the changes in movement pattern seem to reflect those of the bending in the first part of the flagellum, but not in the second part. This is plausible because the second bend, which is the dominant bend, occurs in the first half of the flagellum and frequently in region A-B. These results indicate that the regulation of asymmetry differs in parts of the flagellum, and that the increase in the angle of the dominant bend in the direction of the Rbend affects the movement pattern of hyperactivated spermatozoa.

The differences of regional changes in flagellar bending may be affected by the difference in the cytoskeletal structures between midpiece and principal piece. It is estimated that region $\mathrm{O}-\mathrm{A}$ lies in the midpiece and regions $\mathrm{B}-\mathrm{C}, \mathrm{C}-\mathrm{D}$ and $\mathrm{D}-\mathrm{E}$ lie in the principal piece, as the lengths of midpiece and principal piece are 51 and $126 \mu \mathrm{m}$, respectively (Woolley, 1977). The flexibility, evaluated by changes in the bend angles between two successive frames, changed in a similar manner among regions $\mathrm{B}-\mathrm{C}, \mathrm{C}-\mathrm{D}$ and $\mathrm{D}-\mathrm{E}$ : it decreased with time. However, flexibility changed in a different manner in region $\mathrm{O}-\mathrm{A}$ : it decreased at first, and then increased. The major structural difference between the midpiece and principal piece is that only the principal piece is wrapped by fibrous sheath (Woolley, 1977), which may affect the flexibility of the flagellum.

Temporal examinations revealed that the parameters used to quantify movement pattern and flagellar bending changed in a complex manner. Some parameters changed gradually with time, whereas others changed abruptly before the onset of hyperactivation. Based on microscopic observations, Yanagimachi (1970) reported that hyperactivation occurred abruptly between 2 and $4 \mathrm{~h}$ of incubation. Other reports have described hyperactivated spermatozoa as exhibiting a movement pattern alternating between hyperactive and non-hyperactive phases (Johnson et al., 1981; Tessler and Olds-Clarke, 1985). These observations indicate that hyperactivation is regulated by an on-off switching mechanism (Ho and Suarez, 2001). In the present study, some movement parameters changed abruptly at the onset of hyperactivation, consistent with previous descriptions. VCL, VAP and STR increased abruptly between 2 and $4 \mathrm{~h}$, whereas LIN, ALH, beat cross frequency and average wavelength changed gradually with time. In the analysis of flagellar bending, the asymmetry gradually increased in the first half of the flagellum, whereas the flexibility of the first half of the flagellum abruptly decreased between $10 \mathrm{~min}$ and $1 \mathrm{~h}$, and then increased between 2 and $4 \mathrm{~h}$. These results indicate that complex physiological changes occur before hyperactivation, and that hyperactivation is not controlled simply by an on-off mechanism, but by multiple mechanisms under complex control. 
Hyperactivation has not been defined using quantitative measurements of sperm movement or flagellar bending. Although many studies have measured several parameters, they have showed only that the values of the parameters changed after hyperactivation occurred (Ho and Suarez, 2001). Furthermore, few reports explain how the changes in such parameters are responsible for the change in motility with hyperactivation. In the present study, the change in various parameters was measured with time, and it was determined that the parameters changed in various manners. From microscopic observations, however, hyperactivation appeared to occur abruptly, in agreement with previous descriptions (Yanagimachi, 1970). Two parameters of flagellar bending should be considered to explain which parameters are related to hyperactivation and how the changes in such parameters are responsible for the change to hyperactivated movement. First, the asymmetry of the direction of the R-bend gradually increased in the first half of the flagellum with time. Second, flexibility abruptly decreased between $10 \mathrm{~min}$ and $1 \mathrm{~h}$ and abruptly increased between 2 and $4 \mathrm{~h}$. As it has been reported that hyperactive motility involves asymmetric movement and an elastic flagellum (Ho and Suarez, 2001), it is postulated that the synergic effects of the increase in asymmetry and flexibility at between 2 and $4 \mathrm{~h}$ give the impression of an abrupt change in motility pattern.

The present study carried out a quantitative analysis of the changes in movement pattern and flagellar bending in sperm preparation in which the depth was about $32 \mu \mathrm{m}$. In this condition, the rotation of spermatozoa was minimized and the flagellum moved in nearly two dimensions. It is difficult to analyse correctly the flagellar bending in a deep chamber, as flagella move in three dimensions and there would be considerable scope for error. Although motility pattern differs depending on the depth of the chamber, it changes in the same time course in any depth of chamber (Shivaji et al., 1995). In the present study, the motility pattern may differ from the freely swimming pattern in a deep chamber, but the changes in motility pattern observed reflect those that occur in freely swimming spermatozoa.

The mechanism regulating hyperactivation does not seem to be simple. In the analysis of flagellar bending, asymmetry in the direction of the R-bend gradually increased in the first half of the flagellum with time, whereas flexibility abruptly decreased between $10 \mathrm{~min}$ and $1 \mathrm{~h}$ and abruptly increased between 2 and $4 \mathrm{~h}$. These results indicate that at least two factors are involved in the mechanism producing the change in motility pattern during the course of hyperactivation. A candidate for the factor responsible for the gradual change is A-kinase anchoring protein. The phosphorylation of its tyrosine residues gradually increases with time in hamster spermatozoa (Kula Nand and Shivaji, 2002). Although it is localized to the fibrous sheath in the principal piece
(Carrera et al., 1994; Moss et al., 1999; Kula Nand and Shivaji, 2002) and only a part of this region lies within the first half of flagellum where the gradual change occurs, the phosphorylation of tyrosine residues may be involved in the regulation of the waveform in the upstream region. It remains unclear what is responsible for the abrupt change.

The present study demonstrates that parameters related to patterns of movement and flagellar bending change in various manners with time before hyperactivation. The results promote the hypothesis that multiple factors are involved in the regulation of hyperactivation. The mechanism regulating the asymmetry and flexibility of flagellar bending is especially important.

The authors thank M. Okuno of Tokyo University for helpful discussion and encouragement.

\section{References}

Aoki F, Ishida K, Okuno M and Kohmoto K (1994) Analysis of flagellar bending in hyperactivated hamster and mouse spermatozoa Journal of Reproduction and Fertility 101 397-403

Cancel AM, Lobdell D, Mendola P and Perreault SD (2000) Objective evaluation of hyperactivated motility in rat spermatozoa using computerassisted sperm analysis Human Reproduction 15 1322-1328

Carrera A, Gerton GL and Moss SB (1994) The major fibrous sheath polypeptide of mouse sperm: structural and functional similarities to the A-kinase anchoring proteins Developmental Biology 165 272-284

Ho HC and Suarez SS (2001) Hyperactivation of mammalian spermatozoa: function and regulation Reproduction 122 519-526

Ishijima S, Baba S, Mohri H and Suarez SS (2002) Quantitative analysis of flagellar movement in hyperactivated and acrosome-reacted golden hamster spermatozoa Molecular Reproduction and Development 61 376-384

Johnson LL, Katz DF and Overstreet JW (1981) The movement characteristics of rabbit spermatozoa before and after activation Gamete Research 4 275-282

Kula Nand J and Shivaji S (2002) Identification of the major tyrosine phosphorylated protein of capacitated hamster spermatozoa as a homologue of mammalian sperm A-kinase anchoring protein Molecular Reproduction and Development $61258-270$

Lindemann CB, Gardner TK, Westbrook E and Kanous KS (1991) The calcium-induced curvature reversal of rat sperm is potentiated by CAMP and inhibited by anti-calmodulin Cell Motility and the Cytoskeleton 20 316-324

Mortimer ST and Maxwell WM (1999) Kinematic definition of ram sperm hyperactivation Reproduction, Fertility and Development 11 25-30

Mortimer ST and Swan MA (1995) Kinematics of capacitating human spermatozoa analysed at $60 \mathrm{~Hz}$ Human Reproduction $10873-879$

Moss SB, Turner RM, Burkert KL, VanScoy Butt $\mathbf{H}$ and Gerton GL (1999) Conservation and function of a bovine sperm A-kinase anchor protein homologous to mouse AKAP82 Biology of Reproduction 61 335-342

Neill JM and Olds-Clarke P (1987) A computer-assisted assay for mouse sperm hyperactivation demonstrates that bicarbonate but not bovine serum albumin is required Gamete Research 18 121-140

Ren D, Navarro B, Perez G, Jackson AC, Hsu S, Shi Q, Tilly JL and Clapham DE (2001) A sperm ion channel required for sperm motility and male fertility Nature 413 603-609

Shingyoji C, Murakami A and Takahashi K (1977) Local reactivation of Triton-extracted flagella by iontophoretic application of ATP Nature $\mathbf{2 6 5}$ 269-270 
Shivaji S, Peedicayil J and Girija Devi L (1995) Analysis of the motility parameters of in vitro hyperactivated hamster spermatozoa Molecular Reproduction and Development 42 233-247

Suarez SS (1988) Hamster sperm motility transformation during development of hyperactivation in vitro and epididymal maturation Gamete Research 19 51-65

Tessler S and Olds-Clarke P (1985) Linear and nonlinear mouse sperm motility patterns. A quantitative classification Journal of Andrology 6 35-44

White DR and Aitken RJ (1989) Relationship between calcium, cyclic AMP, ATP and intracellular $\mathrm{pH}$ and the capacity of hamster spermatozoa to express hyperactivated motility Gamete Research 22 163-177

Wiesner B, Weiner J, Middendorff R, Hagen V, Kaupp UB and Weyand I (1998) Cyclic nucleotide-gated channels on the flagellum control $\mathrm{Ca}^{2+}$ entry into sperm Journal of Cell Biology 142 473-484

Woolley DM (1977) Evidence for 'twisted plane' undulations in golden hamster sperm tails Journal of Cell Biology 75 851-865
Yanagimachi R (1970) The movement of golden hamster spermatozoa before and after capacitation Journal of Reproduction and Fertility $\mathbf{2 3}$ 193-196

Yanagimachi R (1981) Mechanisms of fertilization in mammals. In Fertilization and Embryonic Development In Vitro pp 81-182 Eds L Mastroianni and JD Biggers. Plenum Press, New York

Yanagimachi R (1994) Mammalian fertilization. In The Physiology of Reproduction pp 189-317 Eds E Knobil and JD Neill. Raven Press, New York

Received 27 June 2002.

First decision 2 September 2002

Revised manuscript received 11 September 2002.

Accepted 19 September 2002. 